Full metadata for this item is available in Research@StAndrews:FullText at: http://research-repository.st-andrews.ac.uk/

\title{
Calibration of the torsional and lateral spring constants of cantilever sensors
}

\author{
John D. Parkin and Georg Hähner
}

\begin{tabular}{|l|l|}
\hline Date of deposit & 28052014 \\
\hline Version & This is an author version of this work. \\
\hline Access rights & $\begin{array}{l}\text { C This item is protected by original copyright. } \\
\text { This work is made available online in accordance with publisher } \\
\text { policies. To see the final definitive version of this paper please } \\
\text { visit the publisher's website. }\end{array}$ \\
\hline $\begin{array}{l}\text { Citation for } \\
\text { published version }\end{array}$ & $\begin{array}{l}\text { Parkin, J. D., and Hähner, G. (2014). Calibration of the torsional } \\
\text { and lateral spring constants of cantilever sensors. } \\
\text { Nanotechnology, 25(22): 225701 }\end{array}$ \\
\hline $\begin{array}{l}\text { Link to published } \\
\text { version }\end{array}$ & http://dx.doi.org/10.1088/0957-4484/25/22/225701 \\
\hline
\end{tabular}




\title{
Calibration of the torsional and lateral spring constants of cantilever sensors
}

\author{
John D Parkin and Georg Hähner \\ EaStCHEM School of Chemistry, University of St. Andrews, North Haugh, \\ St. Andrews, KY16 9ST, UK \\ "fax: +44 1334463808 phone: +441334463889 E-mail: gh23@st-andrews.ac.uk
}

\begin{abstract}
A method suitable for the calibration of the spring constants of all torsional and lateral eigenmodes of micro- and nanocantilever sensors is described. Such sensors enable nanomechanical measurements and the characterization of nanomaterials, for example with atomic force microscopy.

The method presented involves the interaction of a flow of fluid from a microchannel with the cantilever beam. Forces imparted by the flow cause the cantilever to bend and induce a measurable change of the torsional and lateral resonance frequencies. From the frequency shifts the cantilever spring constants can be determined. The method does not involve physical contact between the cantilever or its tip and a hard surface. As such it is noninvasive and does not risk damage to the cantilever.

Experimental data is presented for two rectangular microcantilevers with fundamental flexural spring constants of 0.046 and $0.154 \mathrm{~N} / \mathrm{m}$. The experimentally determined torsional stiffness values are compared with those obtained by the Sader method. We demonstrate that the torsional spring constants can be readily calibrated using the method with an accuracy of around $15 \%$.
\end{abstract}




\section{Introduction}

Micro- and nanocantilevers are increasingly used in micro- and nanoelectromechanical systems (MEMS and NEMS), for example as pressure and acceleration sensors, linear actuators, or valves [1]. Microcantilevers in particular are employed as probes in atomic force microscopy (AFM) to image surface properties [2]. They have also been applied as independent sensors for mass [3], surface stress [4], chemical identification [4], viscosity and density of liquids [5, 6], or for measuring viscoelastic properties of cells [7]. Small changes in the oscillation properties of the eigenmodes of the cantilevers can be exploited to quantify various physical properties.

Most of the current AFM applications make use of the flexural deflection of the cantilever sensor. In conjunction with mapping of friction forces [8], however, the static torsional deflection of the cantilever is monitored and the related spring constant is required for quantification. The dynamic properties of a torsional eigenmode can be exploited in frequency modulated AFM, where the tip probes forces parallel to the surface with high resolution while scanning [9-11]. Similar resolution has been displayed by images collected using tapping mode and torsional resonance AFM [12]. Images using the oscillation of the true lateral mode have also been collected [10]. The higher quality factor of torsional compared to flexural oscillations in liquid can be utilized to collect images of samples where imaging with the fundamental flexural mode is problematic [13]. If measurements involving lateral or torsional deflections shall be quantified then the associated spring constants must be calibrated [14]. Methods to calibrate the lateral and torsional deflections may be split into three categories: static, dynamic and theoretical. In the former, stationary bending of the cantilever is monitored during physical contact between the cantilever or tip and a calibration sample to produce a lateral/torsional force and therefore involve a high risk of damage. The lateral force can be applied by moving the tip sideways into a step on a calibration grid [15, $16]$, by scanning the cantilever up and down slopes of a known angle on a calibration sample [17-20], or by bringing the tip of the cantilever to be calibrated into contact with another calibrated cantilever that is rotated 90 degrees with respect to the first such that its flexural deformation causes a torsional twist in the first cantilever [21]. Loads could also be applied directly to the cantilever surface, with the resulting cantilever twist being monitored [22, 23]. Methods to calibrate cantilevers by optical means also exist. Whilst these methods do not risk damage to the cantilever, they are sensitive to detector non-linearity [24-26] or rely on the tilting of a calibration surface, which may have a different reflectivity to that of the cantilever [27-29].

Dynamic calibration methods monitor the cantilever's resonance frequency as a pathway to analyzing the interaction of the cantilever with external influences or its response to masses attached to the cantilever [30]. The torsional Cleveland method allows for the determination 
of torsional spring constants by on-axis loading of masses on the cantilever surface [30]. The torsional Sader method determines the torsional spring constant of cantilevers via their interaction with a surrounding (resting) fluid [30]. Following the determination of a 'hydrodynamic function' for a cantilever of a certain shape, all similar cantilevers can be calibrated, provided the cantilever length is much greater than its width and its thickness.

The spring constants of a cantilever may also be calculated theoretically. Analytical equations can be used along with knowledge of the cantilever dimensions and elastic properties to calculate the spring constants [31]. However, large errors (30-50\%) are introduced via assumptions on the elastic constants and in the measurement of the cantilever thickness. Analytical equations can only be used for simple geometries. For more complicated cantilever beams, finite element simulations can be used to determine the spring constants [32].

Simple, non-destructive methods for the calibration of the torsional and lateral stiffness of microcantilevers are highly desirable. In this document we describe a new method that can be utilized to calibrate the torsional and lateral spring constants of cantilever beams in general and is applied to microcantilevers in particular.

\section{Method}

The operating principle of our method is to apply external forces that change the static cantilever shape, but which do not affect the free torsional and lateral movement of the cantilever for example by causing friction or damping. Figure 1 displays the side-view of a straight cantilever and a cantilever that is bent due to external forces.

a)

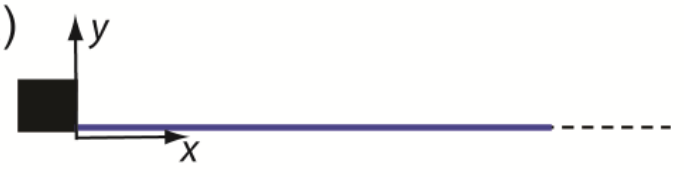

b)

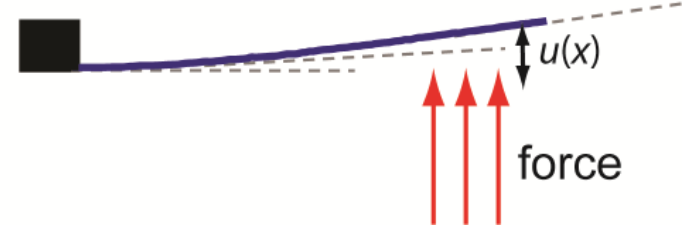

Figure 1: Side view of a non-bent cantilever (a) and a cantilever bent due to forces (b). The dashed lines indicate the rotational axes around which the free torsion occurs. The axis changes along the cantilever if the cantilever is curved.

For the unbent cantilever the centroid of the cantilever coincides with its shear axis. For the bent shape $u(x)$ (figure $1 \mathrm{~b})$ ) the centroid of the cantilever is at a distance, $c(x)$, from the shear-center [33]. Depending on the shape change the torsional modes couple to the lateral 
and/or flexural modes when the cantilever oscillates [33]. If the deflection is entirely in the direction of the flexural modes ( $y$-direction in figure 1) then the torsional oscillations will couple to the lateral oscillations (in the $z$-direction) only. The effective distance $c(x)$ between the centroid and the shear axis can be obtained by weighting $\Delta_{x} u\left(x^{\prime}\right)$, which is the distance of the position of the cantilever at coordinate $x$ to the tangent to the cantilever in $x^{\prime}$, with the angle $d \varphi$ that the cantilever structure at position $x$ rotates around the tangent in $x^{\prime}$. This leads to:

$$
c(x) \approx \frac{\int_{0}^{x} \Delta_{x} u\left(x^{\prime}\right)^{2} d x^{\prime}}{\int_{0}^{x} \Delta_{x} u\left(x^{\prime}\right) d x^{\prime}},
$$

provided $(d u / d x)^{2}<<1$.

The following differential equations for the position dependent part of the coupled torsional and lateral vibrations are obtained if no time dependent external torque or forces are applied [33]:

$$
\begin{gathered}
G K \frac{\partial^{2} \gamma(x)}{\partial x^{2}}-\rho_{c} I_{p} \omega^{2} \gamma(x)+\rho_{c} A(x) c(x) \omega^{2}(Z(x)-c(x) \gamma(x))=0 \\
\frac{\partial^{2}}{\partial x^{2}}\left(E I_{y} \frac{\partial^{2} Z}{\partial x^{2}}\right)-\rho_{c} A(x) \omega^{2}(Z(x)-c(x) \gamma(x))=0
\end{gathered}
$$

where $G$ is the shear modulus, $K$ is a geometric function of the cross section [34], $\gamma(x)$ is the torsional angular deviation from the equilibrium position along the cantilever when oscillating, $\rho_{c}$ is the density, $I_{p}$ the polar moment of inertia about the centroid [34], $A(x)$ is the cross section of the cantilever, $E$ is Young's modulus, $I_{y}$ is the moment of inertia around the $y$-axis, $Z(x)$ is the lateral deflection along the cantilever when oscillating, and $\omega$ is the radial frequency of oscillation.

The lateral and torsional eigenmode shapes of non-bent rectangular cantilevers, $Z_{m}(x)$ and $\gamma_{n}(x)$, respectively, can be obtained analytically [33]. The eigenmodes of many nonrectangular cantilevers for both flexural and torsional vibrations can be determined 
numerically for example by using the Ritz method [33, 35]. Note that the uncalibrated torsional modal shapes $\gamma_{n}(x)$ and the lateral mode shapes $Z_{m}(x)$ are entirely determined by the geometry of the cantilever via the dependence of the parameters $G, K, I_{p}$, and $\rho_{c}$ on $x$ (geometry) in case of $\gamma_{\mathrm{n}}(x)$, and $E, I_{y}$ and $\rho_{c}$ in case of $Z_{\mathrm{m}}(x)$, but not by their absolute values.

Solutions of the differential equations (2a) and (2b) are given by linear superpositions of the functions $\gamma_{n}$ and $Z_{m}$ with coefficients $\alpha_{n}$ and $\beta_{m}$, respectively, which have to be determined. Using the Ritz method this leads to a system of linear equations giving the frequencies $\omega$ and the corresponding eigenmodes of the bent cantilever.

In general the coupling between a torsional and a lateral mode is strongest if their frequency values are 'close'. The torsional modes will therefore in many cases couple to the nearest lateral mode only and vice versa. In these cases it is sufficient to consider the coupling between one torsional mode $\gamma_{n}$ and one lateral mode $Z_{m}$ (although more modes can be taken into account if necessary). Without coupling, two independent values for the undistorted torsional and lateral frequency, $\omega_{\text {tors }}$ and $\omega_{\text {lat }}$, respectively, are obtained. For the coupled oscillations also two values $\omega$ result, that can be determined using the Ritz method [33]:

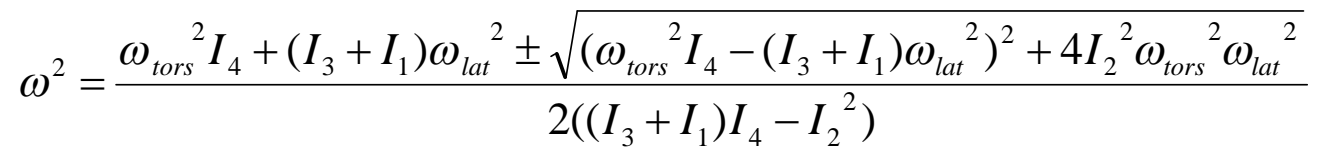

with

$$
\begin{gathered}
I_{1}=\int_{0}^{L} \rho_{c} A c^{2} \gamma_{n}^{2} d x \\
I_{2}=\int_{0}^{L}\left(\int_{c} \rho_{c} A c Z_{m} d \gamma_{n}+\int_{c} \rho_{c} A c \gamma_{n} d Z_{m}\right) d x \\
I_{3}=\int_{0}^{L} \rho_{c} I_{p} \gamma_{n}^{2} d x=\int_{0}^{L} \rho_{c} A \frac{w^{2}}{12} \gamma_{n}^{2} d x \\
I_{4}=\int_{0}^{L} \rho_{c} A Z_{m}^{2} d x
\end{gathered}
$$

where $L$ is the length of the cantilever. The coupling is given by the two integrals $I_{1}$ and $I_{2}$ via $c(x)$ and hence the bent shape $u(x)$. No coupling exists if $u(x)$ and therefore $c(x)$ are zero. For convenience, the modal shapes $\gamma_{n}$ and $Z_{m}$ can be pre-normalized such that $\int_{0}^{L} \frac{w^{2}}{12} \gamma_{n}^{2} d x=1$ 
and $\int_{0}^{L} Z_{m}{ }^{2} d x=1$, where $w$ is the width of the cantilever. Note, however, that the method is independent of the pre-normalization.

For data evaluation a single parameter, (corresponding to $\rho A$ in case of the above described pre-normalization), or two independent parameters, one for $\gamma_{n}$ and one for $Z_{m}$, can be fitted in equation (3), such that the theoretical frequencies match those observed in the experiment. Two parameters are required if the 'pre-normalization' is different from the one above. If the cantilever behaves like an ideal rectangular cantilever a single parameter should reproduce the experimental data well with the above pre-normalization. However, a real cantilever will often display some non-ideal behavior. Fitting two independent parameters can then account for some of the non-ideal behavior with respect to the torsional and lateral oscillations.

The fitting enables the normalization of the eigenmode functions $\gamma_{n}(x)$ and $Z_{m}(x)$ such that:

$$
I_{3}=\int_{0}^{L} \rho_{c} I_{p} \hat{\gamma}_{n}^{2}(x) d x=1
$$

and

$$
I_{4}=\int_{0}^{L} \rho_{c} A \hat{Z}_{m}^{2}(x) d x=1
$$

With the normalized function, $\hat{Z}_{m}$, the true lateral spring constant $k_{\text {lat, } m}$ for a lateral force applied at the free end of the cantilever can be obtained [35]:

$$
k_{l a t, m}=\frac{\omega_{l a t, m}^{2}}{\hat{Z}_{m}^{2}(L)}
$$

A related expression exists to calculate the torsional spring constant $k_{\Phi, n}$ :

$$
k_{\Phi, n}=\frac{\omega_{t o r s, n}^{2}}{\hat{\gamma}_{n}^{2}(L)}
$$




\section{Experimental Details}

\subsection{Setup and dynamic measurements}

Experiments were performed with a commercial AFM Explorer system (ThermoMicroscopes, Sunnyvale, CA, USA). To test the method two commercially available tipless rectangular silicon cantilevers were investigated. Both cantilevers had a nominal width of $35 \pm 3 \mu \mathrm{m}$ and length of $350 \pm 5 \mu \mathrm{m}$. 'Nominal' refers to the information provided by the manufacturer. The first cantilever (Mikromasch), labelled R-E1, had an Al backside coating, a nominal spring constant, $k_{\text {flex }}$, of $0.03 \mathrm{~N} / \mathrm{m}$, and a nominal thickness of $1 \mu \mathrm{m}$. The second (Mikromasch, no backside coating), labelled R-E2, had a nominal spring constant of $0.3 \mathrm{~N} / \mathrm{m}$ and a nominal thickness of $2 \mu \mathrm{m}$. The plan view dimensions of both microcantilevers were also determined with an Olympus optical microscope.

In our setup a homemade smooth parallel plate microchannel of height $100 \mu \mathrm{m}$ and length $3.5 \mathrm{~mm}$ was fixed on the sample stage of the AFM and positioned such that fluid flow from its exit interacted with the cantilever as illustrated in figure 2. The channel was aligned such that the free end of the cantilever was level with the edge of the channel and $50 \mu \mathrm{m}$ above the channel exit.

Nitrogen gas was used as the working fluid. Pressure differences were applied to the microchannel to drive the flow, establishing stable Poiseuille velocity profiles [36]. The maximum pressure applied to the channel in our experiments was $\sim 1.7 \mathrm{kPa}$ causing a velocity value of nitrogen in the channel mid-line of about $35 \mathrm{~m} / \mathrm{s}$ [37]. The highest Reynolds number for our setup did not exceed 200. In combination with the Knudsen number in the channel, which is about 0.0008 , this Reynolds number corresponds to the laminar regime of incompressible flow [38].

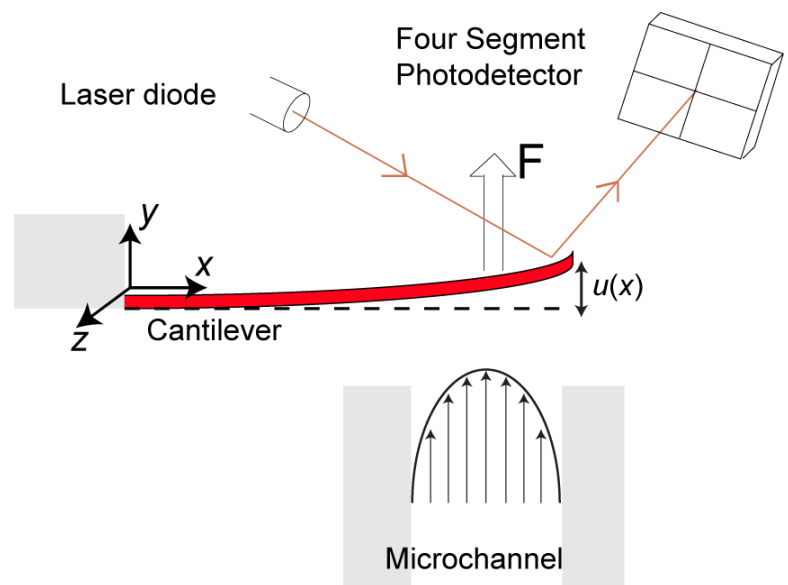

Figure 2: Schematic side view of the microchannel with Poiseuille profile of the fluid and a cantilever that bends due to forces exerted by the fluid. 
The forces applied to the cantilever by the fluid flow cause a static flexural bending, $u(x)$. The bending is sufficient to ensure that the centroid of the cantilever and the shear-center no longer coincide, causing a coupling of the torsional and the lateral oscillation modes and therefore a change in the torsional and lateral resonance frequencies, which can be monitored.

Power spectral densities of thermal noise spectra were recorded with an external interface with an accessible frequency range of $\sim 700 \mathrm{kHz}$ (National Instruments, USB-6251) as a function of fluid velocity. Resonance frequency values, Q-factors, and peak areas for each mode were monitored during measurement with a homewritten LabVIEW ${ }^{\mathrm{TM}}$ routine by fitting Lorentzian curves to the resonance peaks. All resonance frequencies were monitored simultaneously and are based on the average of 50 individual spectra. Note that a standard AFM setup is used where a laser spot is focused on the cantilever and reflected into a photodiode detector. It is the coupling of the torsional mode with the first lateral mode which allows us to observe the resonance curve of the latter: when coupled the initially purely torsional mode takes on a small amount of lateral character and vice versa. Hence, the lateral resonance peak was only observable with fluid flow for the bent cantilever but not without flow (see figure 3).

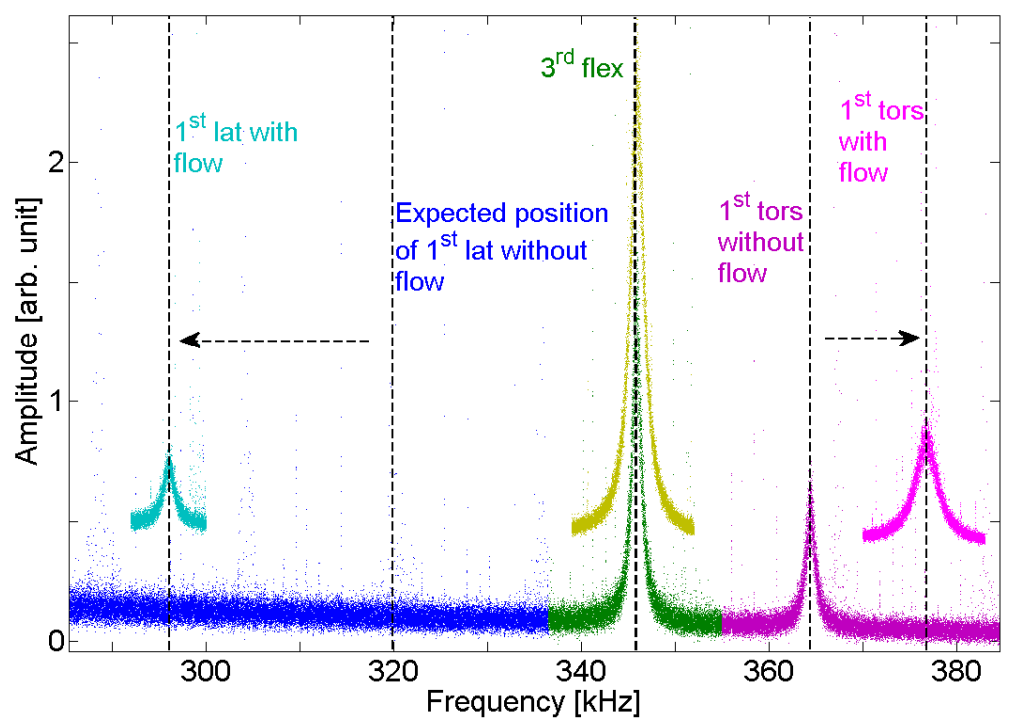

Figure 3: Frequency spectrum of cantilever R-E2 with and without flow, showing the region of the third flexural and fundamental lateral and torsional modes. A constant offset was added to the resonance spectra with flow for reasons of clarity. The arrows indicate the shifts of the lateral and torsional resonance frequencies due to the fluid flow. 


\subsection{Determination of the bent shape $u(x)$ and the spring constants}

To provide information about the interaction of the flow from the channel with the cantilever we performed finite element method simulations with the open-source, multi-physics software package of Elmer [39]. Meshes required in the simulations were created with Gmsh [40]. The mesh independence of the results was confirmed by mesh refinement. The forces applied to the static cantilever by the fluid flow were directly extracted from the simulations. Figure 4 displays the forces applied to $350 \mu \mathrm{m}$ long rectangular microcantilevers (R-E1 and R-E2) with fluid speeds at the center of the microchannel of $6,14,22$ and $30 \mathrm{~m} / \mathrm{s}$.

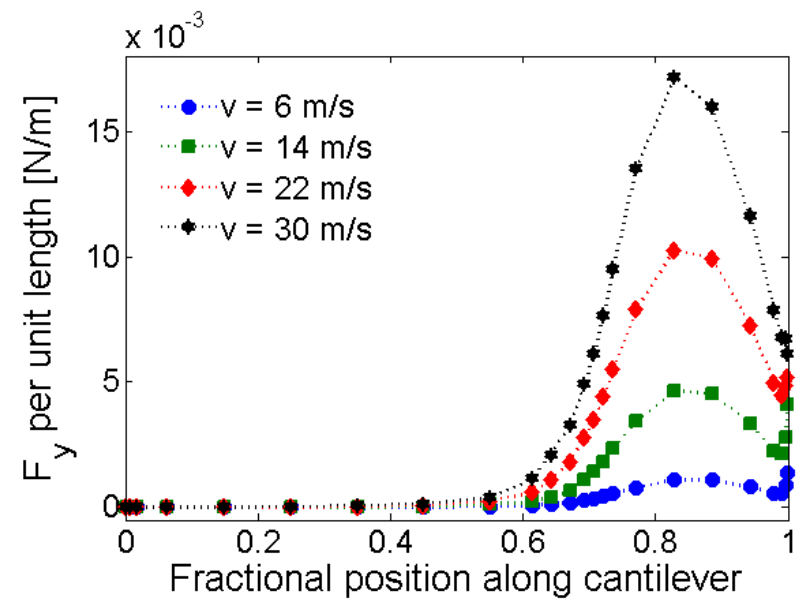

Figure 4: Simulated fluidic forces applied to a rectangular cantilever of $350 \mu \mathrm{m}$ length and $35 \mu \mathrm{m}$ width at speeds of $6,14,22$ and $30 \mathrm{~m} / \mathrm{s}$ in the center of the microchannel

The static deflection $u(x)$ depending on the applied fluidic forces was determined with a home written MATLAB® routine by generalizing the results reported in $[35,41]$ for the force profiles extracted from Elmer and the flexural rigidity of each cantilever. The flexural spring constants were obtained beforehand independently as described in [42]. Note that the static equilibrium bent shape $u(x)$ could also be determined with other methods: for example via interferometry or by taking a photograph if the cantilever and its deflection are large enough. In our experiments the highest value for $(d u / d x)^{2}$ was 0.007 in accordance with the derivation of equation (1). The effective distance $c(x)$ was then determined from equation (1). The experimental frequency data was fitted using equation (3) with both a single as well as two independent parameters and the spring constants determined via equations (6) and (7).

\section{Results}

4.1 Cantilever dimensions and flexural spring constant calibration 
The plan view dimensions of the cantilevers are summarized in table 1. Significant discrepancies from the manufacturer's (nominal) dimensions were found, particularly in relation to the width of the beams. Both cantilevers had a reasonably rounded or picketed free end. The quoted lengths were taken to be at the extreme of the structures. Also shown are the experimentally determined static flexural spring constants $k_{\text {flex }}$, the nominal values quoted by the manufacturers, and 'ideal' values, which were determined theoretically by using the experimentally determined plan view dimensions and the nominal thickness values together with a density of $2330 \mathrm{~kg} / \mathrm{m}^{3}$ and a Young's modulus of $169 \mathrm{GPa}$ [43].

\begin{tabular}{|c|c|c|c|c|c|c|c|c|}
\hline & \multicolumn{2}{|c|}{$\boldsymbol{L}(\boldsymbol{\mu m})$} & \multicolumn{2}{c|}{$\boldsymbol{w}(\boldsymbol{\mu m})$} & $\boldsymbol{t}(\boldsymbol{\mu m})$ & \multicolumn{3}{c|}{$\boldsymbol{k}_{\text {flex }}(\mathbf{N} / \mathbf{m})$} \\
\hline & Exp. & Nom. & Exp. & Nom. & Nom. & Exp. & Ideal & Nom. \\
\hline R-E1 & 340 & 350 & 30.7 & 35 & 1 & 0.046 & 0.034 & 0.03 \\
\hline R-E2 & 341 & 350 & 31.0 & 35 & 2 & 0.154 & 0.273 & 0.3 \\
\hline
\end{tabular}

Table 1: Plan view dimensions and static flexural spring constants of the cantilevers tested.

\subsection{Frequency shifts}

Table 2 summarizes the experimental flexural, torsional and lateral frequency values of the non-bent cantilevers and the values for the ideal cantilevers obtained from calculation with the measured plan view dimensions. A shear modulus of $51 \mathrm{GPa}$ was used [43].

The experimental lateral frequencies $f_{0, \text { lat }}$ without flow were extrapolated from the values obtained with flow, with an estimated uncertainty in the frequency of less than $5 \%$. The ideal torsional and lateral oscillation frequencies show some deviation from the experimentally determined ones, indicating that the cantilevers behave non-ideally.

\begin{tabular}{|l|c|c|c|c|c|c|}
\hline & \multicolumn{2}{|c|}{$\boldsymbol{f}_{\text {flex }}(\mathbf{k H z})$} & \multicolumn{2}{c|}{$\boldsymbol{f}_{\text {tors }}(\mathbf{k H z})$} & \multicolumn{2}{c|}{$\boldsymbol{f}_{\text {lat }}(\mathbf{k H z})$} \\
\hline & Exp & Ideal & Exp & Ideal & Exp & Ideal \\
\hline R-E1 & 11.9 & 11.8 & 247 & 222 & $' 308 '$ & 365 \\
\hline R-E2 & 17.1 & 23.7 & 358 & 443 & $' 320 '$ & 367 \\
\hline
\end{tabular}

Table 2: Flexural, torsional and lateral frequencies of the first dynamic modes of the cantilevers tested. 
Model calculations were performed to solve equations (2) numerically with a home-written MATLAB® routine for the ideal cantilevers taking the first two torsional and the first two lateral modes into account. Table 3 summarizes the contributions of the 'pure' uncoupled modes to the coupled oscillations. The contributions were calculated for deflections of $1 \mu \mathrm{m}$ and $3.75 \mu \mathrm{m}$ at the free end of each cantilever, which are typical values in our experiment. Note that the absolute values of the contributions depend on the deflection, but the ratio of the contributing non-major modes is rather similar.

\begin{tabular}{|c|c|c|c|c|c|c|}
\hline & Modes & Defl $(\mu \mathrm{m})$ & $1^{\text {st }}$ tors $(\%)$ & $2^{\text {nd }}$ tors (\%) & $1^{\text {st }}$ lat (\%) & $2^{\text {nd }}$ lat (\%) \\
\hline \multirow{4}{*}{ R-E1 } & \multirow{2}{*}{$1^{\text {st }}$ tors coupled } & 1 & 99.59 & 0.00 & 0.41 & 0.00 \\
\hline & & 3.75 & 94.94 & 0.05 & 4.99 & 0.02 \\
\hline & \multirow{2}{*}{$1^{\text {st }}$ lat coupled } & 1 & 0.06 & 0.10 & 99.84 & 0.00 \\
\hline & & 3.75 & 0.69 & 1.46 & 97.85 & 0.00 \\
\hline \multirow{4}{*}{ R-E2 } & \multirow{2}{*}{$1^{\text {st }}$ tors coupled } & 1 & 99.19 & 0.00 & 0.81 & 0.00 \\
\hline & & 3.75 & 91.17 & 0.07 & 8.74 & 0.02 \\
\hline & \multirow{2}{*}{$1^{\text {st }}$ lat coupled } & 1 & 1.69 & 0.06 & 98.25 & 0.00 \\
\hline & & 3.75 & 16.70 & 0.68 & 82.62 & 0.00 \\
\hline
\end{tabular}

Table 3: Percentage contributions of the uncoupled modes to the coupled oscillations for deflections of $1 \mu \mathrm{m}$ and $3.75 \mu \mathrm{m}$ at the cantilevers free end.

Figure 5 shows typical frequency shifts, $\Delta\left(f^{2}\right)=f^{2}-f_{0}^{2}$, where $f_{0}$ is the frequency without flow and $f$ the frequency measured with flow, of the first torsional and lateral resonance frequencies for cantilevers R-E1 and R-E2, depending on the speed of the fluid escaping from the microchannel. Also shown are fits with two independent fit parameters for the torsional and the lateral mode, respectively, based on equation (3). Using a single fit parameter did not produce results of similar quality. In particular for R-E2 fits with a single parameter did not resemble the experimental data well. 

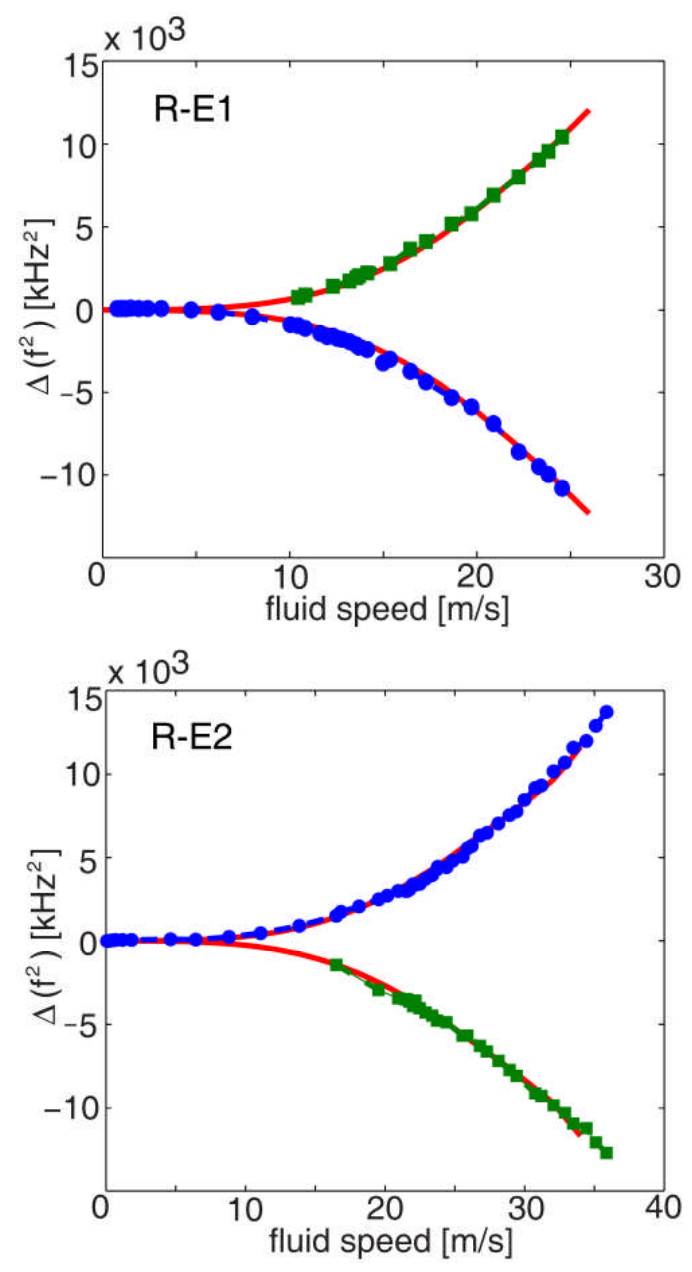

Figure 5: Frequency shifts of the first torsional (blue circles) and first lateral (green squares) eigenmodes of cantilevers R-E1 (top) and R-E2 (bottom) due to static bending caused by fluid flow from a microchannel. The solid red lines show the fit from the model.

\subsection{Torsional and lateral spring constant calibration}

The procedure described above produces values for the dynamic torsional and lateral spring constants. The corresponding static spring constants can be determined from the first dynamic spring constants by dividing the lateral value by 1.03 [35] and the torsional value by $\pi^{2} / 8$. The results are summarized in table 4 together with values obtained by the Sader method [30] and theoretical values for 'ideal' cantilevers. Note that the Sader method is in general not applicable to lateral stiffness [30].

In case of atomic force microscopy the 'lateral' spring constant, $k$, relevant in connection with measuring a lateral force when the scanning tip of an AFM cantilever is in contact with a surface, can be obtained from $k_{\text {tors }}[44]$ via: 


$$
k_{l}=\frac{k_{t o r s}}{h_{t i p}^{2}}
$$

where $h_{\text {tip }}$ is the height of the tip attached at the free end of the AFM cantilever. A lateral force applied to a tip attached at the end of the AFM cantilever causes a torsional twist of the beam, which is measured. This 'lateral' spring constant however is different from the true lateral spring constant that can be obtained from the normalized lateral eigenmode $Z_{1}$ using equation (6). The latter corresponds to the stiffness of the cantilever in relation to true lateral deflections. The resulting values are displayed in table 4. It is difficult to obtain these values experimentally in atomic force microscopy or for microcantilevers, because a lateral movement can in general not be detected with the standard readout system for microcantilevers. A rough estimate can be deduced based on the flexural spring constant and the assumption of an 'ideal' cantilever as has been executed in table 4.

\begin{tabular}{|l||c|c|c||c|c|c|}
\hline \multicolumn{1}{|c||}{} & \multicolumn{3}{c||}{ static $\boldsymbol{k}_{\text {tors }}\left(\mathbf{x} \mathbf{1 0}^{-9} \mathbf{N m}\right)$} & \multicolumn{3}{c|}{ static $\boldsymbol{k}_{\text {lat }}$ ( N/m) } \\
\hline & Our method & Sader [30] & Ideal & Our method & Sader [30] & Ideal \\
\hline R-E1 & 1.7 & 2.1 & 2.0 & 25 & N/A & 32 \\
\hline R-E2 & 3.7 & 3.1 & 8.5 & 57 & N/A & 64 \\
\hline
\end{tabular}

Table 4: Comparison of the torsional and true lateral spring constants calibrated by the method described here, the Sader method, and those of ideal rectangular cantilevers with the experimentally determined plan view dimensions and the manufacturer's nominal thickness and material properties values.

\section{Discussion}

\subsection{Coupling of modes and the number of modes required}

The procedure presented exploits the coupling between torsional and lateral modes of oscillation to determine the torsional and lateral stiffness of cantilevers. Coupling between lateral and torsional modes can be observed in AFM [45], or for free cantilevers where there is off-axis mass, e.g. if the tip is displaced from the cantilever center line. In that case the non-symmetrical mass distribution is the origin of the coupling. For the calibration method described here, the static bending of the cantilever breaks the symmetry of the cantilever to induce coupling of the torsional and lateral eigenmodes.

When coupled, the formerly pure torsional eigenmode takes on a small lateral movement and the formerly pure lateral eigenmode involves a component of torsional movement (see table 3). The magnitude of the coupling of one mode to another is in general related to their 
separation in frequency space. If the first lateral resonance is higher than the first torsional frequency then as the cantilever is bent the lateral frequency will increase and the torsional will decrease, as was observed for cantilever R-E1. Opposite behavior is expected if the lateral frequency is lower than the torsional frequency as was the case for cantilever R-E2. There are some cases where this simple rule might not be valid and others where higher eigenmodes must be considered. The model calculations performed for the ideal cantilevers taking into account a higher number of torsional and lateral modes indicate that considering only two modes is sufficient when determining the torsional spring constants of both R-E1 and R-E2, and the lateral spring constant of R-E2 (see table 3). The mainly lateral mode of the bent cantilever R-E1 however has a significant contribution from the second torsional mode, which was not taken into account in the current experimental determination of the lateral spring constant (equation (3)). Part of the deviation for the lateral value of R-E1 from the ideal value might therefore be due to the contribution of the second torsional mode. The influence of the second torsional mode can be understood by the 'closeness' of the frequencies involved: based on the first torsional resonance of R-E1 at $247 \mathrm{kHz}$ the second is expected around $740 \mathrm{kHz}$, while the lateral was found at $308 \mathrm{kHz}$. In contrast, for R-E2 the lateral frequency was at $320 \mathrm{kHz}$, while the first torsional was $357 \mathrm{kHz}$ and the second torsional is expected around $1075 \mathrm{kHz}$.

\subsection{Influence of cantilever thickness}

The thickness of the cantilevers is not known accurately and it is in general difficult to determine it experimentally. It has however a negligible effect on the forces due to the fluid flow from the microchannel experienced by cantilevers R-E1 and R-E2 (figure 4). There was virtually no difference (less than 1\%) in the simulated total force applied to a 1 and $2 \mu \mathrm{m}$ thick cantilever. A deviation from the nominal thickness values might be responsible however for some of the difference between the ideal (based on the nominal thickness) and measured values found for the torsional spring constants (table 4).

\subsection{Single parameter fit versus two parameter fit}

The cantilevers studied deviate from ideal rectangular cantilevers with regard to torsional and lateral behavior as can be seen by the measured frequency values (table 2). It is therefore not surprising that single parameter fits did not reproduce the experimental data with the same quality as those obtained with two parameters. For R-E1 the deviation in the frequency values from the ideal values was relatively small. This could explain why a single parameter fit produced reasonable results for R-E1 but not for R-E2, where the discrepancy was much larger. However, in both cases the experimental data were better reproduced and results more reliable for a two parameter fit. 
It should also be noted that correct torsional and lateral frequency values are crucial if a single parameter is fitted to the experimental data, but not in case of two independent parameters. This is due to the structure of equation (2): Different frequency values will result in correspondingly different fit parameters that are then used for the normalization of the mode shapes. The uncertainty in the lateral frequency without flow therefore does not contribute to the uncertainty in the spring constant determined from equation (6).

\subsection{Spring constant values and influence of uncertainty in bent shape}

The determined torsional spring constants show some deviation from the values obtained with the Sader method (table 4), which is applicable to ideal rectangular cantilevers. The cantilevers used were reasonably picketed and displayed some non-ideal behavior supported by the observed frequency values. In addition, one of the cantilevers (R-E1) had an aluminum back coating that can also contribute to non-ideal behavior and influence the stiffness. This can explain some of the deviation of the results obtained with the method reported here and the Sader method.

The accuracy with which the bent shape of the cantilever is known has a direct influence on the accuracy of the determined spring constant and its uncertainty. All contributing factors have therefore to be considered carefully. The error in the flexural spring constant is estimated to be around 5\% [42]. Positioning of the cantilever relative to the microchannel can be easily achieved within $5 \mu \mathrm{m}$ with our current setup. In addition, there is an uncertainty in the exact force exerted by the fluid flow, partially due to the thickness of the cantilever, but also based on the exact channel dimensions, and the pressure applied to the channel. Based on our simulations and experiments a $5 \mu \mathrm{m}$ deviation from the assumed cantilever position relative to the microchannel, a cantilever thickness variation of $\sim 1 \mu \mathrm{m}$, and a deviation in the fluid flow of $\sim 2 \%$ have a very small influence $(\sim 3 \%)$ on the overall resulting force. We therefore estimate the overall uncertainty in the bent shape to be not more than $\sim 8 \%$. Fitting the experimental data with an $8 \%$ higher and an $8 \%$ lower value of deflection resulted in spring constants with less than $15 \%$ deviation from the reported values. In consequence we estimate the current uncertainty to be $\sim 15 \%$. Note however, that with a more accurate determination of the bent shape this uncertainty could be significantly reduced.

\section{Conclusions}

We have demonstrated that with knowledge of the shape of a cantilever beam and a measure of its resonance frequency in a number of static equilibrium positions, the torsional and lateral spring constants of the cantilever can be determined. The method can be 
performed in situ and does not involve direct contact of the cantilever or tip with another object. This is particularly important when the cantilever or tip have been covered with a sensitive coating or biofilm.

While the tested cantilevers were tipless, the method can also be applied to cantilevers that have a tip or a colloidal probe attached. If the mass of the tip or colloidal particle is significant then this can have an effect on the modal shape and should be taken into account [46].

Whilst coupling between the torsional and lateral modes has been demonstrated, coupling between torsional and flexural modes could similarly be exploited by inducing a static bend in the lateral direction. The method was demonstrated with rectangular microcantilever beams but has the potential to be extended to other cantilever sizes and shapes, e.g. triangular cantilevers. Finally, we note that in cases where the lateral spring constant has been independently calibrated, only the torsional mode needs to be monitored and a single parameter determined for its calibration.

\section{Acknowledgements}

Financial support from the EPSRC (EP/K000411/1) and the University of St. Andrews under an Impact Acceleration Account (EP/K503940/1) are gratefully acknowledged. 


\section{References}

[1] Beeby S, Ensell G, Kraft N and White N 2004 MEMS Mechanical Sensors (London: Artech House )

[2] Giessibl F J 2003 Advances in atomic force microscopy Rev. Mod. Phys. 75 949-83

[3] Parkin J D and Hähner G 2011 Mass determination and sensitivity based on resonance frequency changes of the higher flexural modes of cantilever sensors Rev. Sci. Instrum. 82035108

[4] Boisen A, Dohn S, Keller S S, Schmid S and Tenje M 2011 Cantilever-like micromechanical sensors Rep. Prog. Phys. 74036101

[5] McLoughlin N, Lee S L and Hähner G 2007 Temperature dependence of viscosity and density of viscous liquids determined from thermal noise spectra of uncalibrated atomic force microscope cantilevers Lab Chip 7 1057-61

[6] McLoughlin N, Lee S L and Hähner G 2006 Simultaneous determination of density and viscosity of liquids based on resonance curves of uncalibrated microcantilevers Appl. Phys. Lett. 89184106

[7] Thomson N H, Fritz M, Radmacher M, Cleveland J P, Schmidt C F and Hansma P K 1996 Protein tracking and detection of protein motion using atomic force microscopy Biophys. J. 70 2421-31

[8] Garcia R and Herruzo E T 2012 The emergence of multifrequency force microscopy Nat. Nanotechnol. 7 217-26

[9] Kasai T, Bhushan B, Huang L and Su C M 2004 Topography and phase imaging using the torsional resonance mode Nanotechnology 15 731-42

[10] Reinstadtler M, Kasai T, Rabe U, Bhushan B and Arnold W 2005 Imaging and measurement of elasticity and friction using the TRmode Journal of Physics $D$ Applied Physics 38 R269-R82

[11] Huang L and Su C M 2004 A torsional resonance mode AFM for in-plane tip surface interactions Ultramicroscopy 100 277-85

[12] Turner J A and Wiehn J S 2001 Sensitivity of flexural and torsional vibration modes of atomic force microscope cantilevers to surface stiffness variations Nanotechnology $12322-30$

[13] Mullin N and Hobbs $\mathrm{J} 2008$ Torsional resonance atomic force microscopy in water Appl. Phys. Lett. 92053103

[14] Song $Y X$ and Bhushan B 2005 Quantitative extraction of in-plane surface properties using torsional resonance mode of atomic force microscopy J. Appl. Phys. 97083533

[15] Cannara R J, Eglin M and Carpick R W 2006 Lateral force calibration in atomic force microscopy: A new lateral force calibration method and general guidelines for optimization Rev. Sci. Instrum. 77053701

[16] Choi D, Hwang W and Yoon E 2007 Improved lateral force calibration based on the angle conversion factor in atomic force microscopy J. Microsc.-Oxf. 228 190-9 
[17] Ogletree D F, Carpick R W and Salmeron M 1996 Calibration of frictional forces in atomic force microscopy Rev. Sci. Instrum. 67 3298-306

[18] Varenberg M, Etsion I and Halperin G 2003 An improved wedge calibration method for lateral force in atomic force microscopy Rev. Sci. Instrum. 74 3362-7

[19] Ling X, Butt H J and Kappl M 2007 Quantitative measurement of friction between single microspheres by friction force microscopy Langmuir 23 8392-9

[20] Asay D B and Kim S H 2006 Direct force balance method for atomic force microscopy lateral force calibration Rev. Sci. Instrum. 77043903

[21] Ecke S, Raiteri R, Bonaccurso E, Reiner C, Deiseroth H J and Butt H J 2001 Measuring normal and friction forces acting on individual fine particles Rev. Sci. Instrum. 72 4164-70

[22] Reitsma M G 2007 Lateral force microscope calibration using a modified atomic force microscope cantilever Rev. Sci. Instrum. 78106102

[23] Reitsma M G, Gates R S, Friedman L H and Cook R F 2011 Prototype cantilevers for quantitative lateral force microscopy Rev. Sci. Instrum. 82093706

[24] Cain R G, Reitsma M G, Biggs S and Page N W 2001 Quantitative comparison of three calibration techniques for the lateral force microscope Rev. Sci. Instrum. 72 3304-12

[25] Liu E, Blanpain B and Celis J P 1996 Calibration procedures for frictional measurements with a lateral force microscope Wear 192 141-50

[26] Tocha E, Song J, Schonherr H and Vancso G J 2007 Calibration of friction force signals in atomic force microscopy in liquid media Langmuir 23 7078-82

[27] Feiler A, Attard P and Larson I 2000 Calibration of the torsional spring constant and the lateral photodiode response of frictional force microscopes Rev. Sci. Instrum. 71 2746-50

[28] Bogdanovic G, Meurk A and Rutland M W 2000 Tip friction - torsional spring constant determination Colloid Surf. B-Biointerfaces 19 397-405

[29] Xie H, Vitard J, Haliyo S and Regnier S 2008 Optical lever calibration in atomic force microscope with a mechanical lever Rev. Sci. Instrum. 79096101

[30] Green C P, Lioe H, Cleveland J P, Proksch R, Mulvaney P and Sader J E 2004 Normal and torsional spring constants of atomic force microscope cantilevers Rev. Sci. Instrum. 75 1988-96

[31] Neumeister J M and Ducker W A 1994 Lateral, Normal, and Longitudinal Spring Constants of Atomic-Force Mircroscopy Cantilevers Rev. Sci. Instrum. 65 2527-31

[32] Hazel J L and Tsukruk V V 1998 Friction force microscopy measurements: Normal and torsional spring constants for V-shaped cantilevers J. Tribol.-Trans. ASME 120 814-9

[33] Timoshenko S, Young D H and Weaver Jr. W 1974 Vibration Problems in Engineering (New York: John Wiley ) 
[34] Landau L D and Lifschitz E M 1991 Elastizitätstheorie (Berlin: Akademie Verlag)

[35] Hähner G 2010 Dynamic spring constants for higher flexural modes of cantilever plates with applications to atomic force microscopy Ultramicroscopy $110801-6$

[36] Lubarsky G V and Hähner G 2007 Calibration of the normal spring constant of microcantilevers in a parallel fluid flow Rev. Sci. Instrum. 78095102

[37] Lubarsky G V and Hähner G 2008 Hydrodynamic methods for calibrating the normal spring constant of microcantilevers Nanotechnology 19325707

[38] Hetsroni G, Mosyak A, Pogrebnyak E and Yarin L P 2005 Fluid flow in microchannels Int. J. Heat Mass Transfer 48 1982-98

[39] http://www.csc.fi/elmer

[40] Geuzaine C and Remacle J F 2009 Gmsh: A 3-D finite element mesh generator with built-in pre- and post-processing facilities Int. J. Numer. Methods Eng. 79 1309-31

[41] Hähner G 2008 Normal spring constants of cantilever plates for different load distributions and static deflection with applications to atomic force microscopy J. Appl. Phys. 104084902

[42] Parkin J D and Hähner G 2013 Determination of the spring constants of the higher flexural modes of microcantilever sensors Nanotechnology 24065704

[43] http://www.spmtips.com/afm-probes-faq.afm\#6.

[44] Munz M 2010 Force calibration in lateral force microscopy: a review of the experimental methods Journal of Physics D-Applied Physics 43063001

[45] Song $Y X$ and Bhushan B 2006 Coupling of cantilever lateral bending and torsion in torsional resonance and lateral excitation modes of atomic force microscopy $\mathrm{J}$. Appl. Phys. 99094911

[46] Kiracofe D and Raman A 2010 On eigenmodes, stiffness, and sensitivity of atomic force microscope cantilevers in air versus liquids J. Appl. Phys. 107033506 